\title{
Spectrum Combining Technique at Low SNR for the Frequency Selective Channels
}

\author{
C.Y. Shen, Y.P. Hu, X. Zhang, Y.Q. Tang \\ Information Science and Technology Institute \\ Zhengzhou Henan P.R China
}

\begin{abstract}
To decrease the Inter-Symbol Interference (ISI) for the frequency selective channel at low SNR, a spectrum combining technique based on average phase from the multiple sensors without synchronization is proposed. The combining signal's phase will converge to the average phase instead of the value of the fixed branch to meliorate adverse effect on the demodulation performance caused by the single nonlinear phase characteristic. Finally the simulations show that the spectrum combining technique can achieve a substantial performance improvement without the symbol timing synchronization. Therefore it could be regarded as a useful combining technique in the frequency selective channels at low SNR, especially when synchronization is difficult to achieve.
\end{abstract}

Keywords-frequency selective channel; spectrum combining; multiple sensors; spatial diversity; Average Phase

\section{INTRODUCTION}

The primary challenge of the broadband wireless channel is that the signal is corrupted by the multi-path propagation, and the system performance degrades due to inter-symbol interference (ISI) existing in data transmission for the frequency-selective fading channel [1].To overcome the channel frequency-selective fading and ensure the quality of communication, the combination with space diversity reception and adaptive equalization is chosen as an effective scheme. Therefore the various combiner-equalizer receiver structures are studied in the time domain and the frequency domain [2-6]. Optimum diversity combining and equalization dealing with linear or decision-feedback time equalizers has been studied in many papers [2-4]. On the other hand, space diversity in conjunction with FDE also has been explored in [5-6].

In previous simulations and analyses, it was often supposed that receiver and combiner-equalizer receiver were ideal synchronization or the training sequence was also used for the pilot in synchronization and channel estimation. Hence the system models discard the issue of the time recovery in the diversity and equalization system. In fact, the synchronization is impossible to implement without the accurate known information such as symbol rate and carrier frequency at low SNR for the frequency selective fading channels, or the performance degrades badly for the probability of phase locked loop(PLL) lock loss [7] and it causes the negative effect on the equalization.

Therefore a feasible method to improve the SNR and counteract the frequency selective fading is to combine the signals before synchronization of the branch signal. When the signals are only corrupted by additive Gaussian white noise, the best performance can be obtained by weighting and co-phasing all received signals according to the MRC (Maximum Ratio Combining) criterion. However the signal for each branch is distorted differently for the frequency selective fading channel, it is impossible to co-phase the signal with the constant coefficients and improper to combine all received signals in the time-domain.

In this paper, a spectrum combining technique without synchronization is proposed at low SNR for the frequency selective fading channels, and then the combining signal is synchronized and equalized. In addition, on the basis of analysis on the general demodulation with blind Baud Spaced Equalizer after symbol timing synchronization, a simple average phase algorithm is also demonstrated, and the combining signal's phase can be converged to the average phase instead of the value of the fixed branch. Finally the simulations prove the algorithm's feasibility and performance.

\section{SignAL MODEL}

Without loss of generality, it is supposed that there are L received sensors in the array, with Gaussian noise and intersymbol interference, the base-band signal for each branch can be written as:

$$
x_{i}(t)=\sum_{n} a_{n} h_{i}(t-n T) e^{j 2 \pi \Delta f t}+n_{i}(t)
$$

where $h_{i}(t)=g_{T}(t) \otimes c_{i}(t), i=0, \cdots, L-1 . g_{T}(t)$ is the impulse response of transmitted filter and $c_{i}(t)$ is the ith complex impulse response of the multi-path channel, “ $\otimes$ " denotes convolution. $\left\{a_{n}\right\}$ is a sequence of the uncorrelated random symbols, $\mathrm{T}$ is the symbol period and $\Delta f$ is the carrier frequency offset in the down conversion while the initial arbitrary phase $^{\theta_{i}}$ is included in the $c_{i}(t)$. The component $n_{i}(t)$ can be considered as the independent additive white Gaussian noise and uncorrelated with the received signal each other.

The parameter $c_{i}(t)$ is an invariable constant in the nonfrequency selective channel, and the ith combining weight $w_{i}=c_{i}^{*} e^{j \theta_{c}} / N_{0 i}$ is also constant [8], where ${ }^{N_{0 i}}$ is the noise power density and $\theta_{c}$ is the random combined phase. In the other hand, the signal components in the frequency domain are provided with the different amplitude gain and phase deflexion. 
Consequently, it is improper to sum the signal with the constant weight and unable to achieve the best performance.

\section{SPeCTRUM COMBINING TECHNIQUE}

With Fourier Transformed (FT), each signal denoted by equation (1) can be expressed as following in the frequency domain

$$
X_{i}(\omega)=C_{i}(\omega) S_{l}(\omega)+N_{i}(\omega)
$$

where $C_{i}(\omega), S_{l}(\omega)$ and $N_{i}(\omega)$ denote Fourier Transformed of the ith channel, the signal component and the ith noise respectively.

For a certain frequency $\omega_{k}$, the frequency signal component $X_{i}\left(\omega_{k}\right)$ can be regarded as the $S_{l}\left(\omega_{k}\right)$ multiplied by the channel gain $C_{i}\left(\omega_{k}\right)$ corrupted by the additive white Gaussian noise. Therefore it is appropriately supposed that the $S_{l}\left(\omega_{k}\right)$ goes through a Gaussian channel. As a result, if making use of the mth signal as reference, the ith combining weight[9]

$$
w_{i}\left(\omega_{k}\right)=\left|C_{i}\left(\omega_{k}\right)\right| e^{j \Delta \varphi_{i m}\left(\omega_{k}\right)} / N_{0 i}
$$

$\theta_{i m}\left(\omega_{k}\right)=\varphi_{i}\left(\omega_{k}\right)-\varphi_{m}\left(\omega_{k}\right)$ represents the phase difference of the $C_{i}\left(\omega_{k}\right)$ and $C_{m}\left(\omega_{k}\right)$ at the frequency $\omega_{k}$, and it is easy to achieve the phase value utilizing the correlation between the $m$ th and the $i$ th signal in the frequency domain. It is sure that the SNR of the combined signal becomes greater than the single's. The combining noise power density $N_{0 c}$ is

$$
N_{0 c}(\omega)=\sum_{i=0}^{L-1}\left|C_{i}(\omega)\right|^{2} / N_{0 i}
$$

It can be seen clearly that the combining noise is colored, and it is necessary to restrict the weight amplitude in order to avoid the adverse effect of the colored noise. The modified combining weight is

$$
\hat{w}_{i}(\omega)=\mu w_{i}(\omega) / \sqrt{\sum_{i=0}^{L-1} \frac{1}{N_{0 i}}\left|C_{i}(\omega)\right|^{2}}
$$

where ${ }^{\mu}$ is a constant, and the denominator takes an important role in the whitening noise. It is well known that Fast Fourier Transform (FFT) is usually to transform the signal in the domain to the frequency domain. Each of these signals after is weighted with the parametric $\hat{w}_{i}(k)$ which is estimated making use of the received data, and then combined for all of the branch. Finally the combining result goes through the IFFT, and the combined signal in the time domain is obtained.

\section{AVERAge Phase AlgorithM}

The phase of the combined channel is the same as the reference channel using the algorithm above. But in fact the demodulation scheme with Baud Spaced Equalizer after symbol timing synchronization is often used on the assumption of the unknown channel. As is now well known that the system performance degrades because of the channel's nonlinear phase frequency characteristic. Therefore the combining performance loss will probably occur which is brought by the changeless reference channel, thus it is improper to make use of a certain single channel as reference to co-phase the phase difference.

\section{A. Absolute Average Phase}

To meliorate adverse effect on the combining performance caused by the single reference channel, the new phase compensating algorithm utilizes all of the channels from the array, That is

$$
\varphi^{c}(\omega)=\frac{1}{L} \sum_{i=0}^{L-1} \varphi_{i}(\omega)
$$

It can be seen that not only the $\varphi^{c}(\omega)$ is the average value of the phase of all the branches, and the group delay of the combined is also brought into alignment with the average group delay

$$
\tau^{c}(\omega)=\frac{1}{L} \sum_{i=0}^{L-1} \tau_{i}(\omega)
$$

where $\tau^{c}(\omega)$ and $\tau_{i}(\omega)$ denote the group delay of the combined and the ith channel respectively. Obviously, the combined phase frequency characteristic is linear with when $L \rightarrow \infty$. As a result, the method could improve the characteristic of the phase frequency and overcome the shortcoming from the fixed single method. The compensating phase $\theta_{i}^{c}(\omega)$ of the ith branch is

$$
\theta_{i}^{c}(\omega)=\frac{1}{L} \sum_{j=0, j \neq i}^{L-1}\left[\varphi_{j}(\omega)-\varphi_{i}(\omega)\right]=\frac{1}{L} \sum_{j=0, j \neq i}^{L-1} \theta_{j i}(\omega)
$$

It means that the compensating phase value is equal to the average of the phase difference between the whole signal and the ith branch, and it is easy to estimate the phase value using the correlation.

\section{B. Relative Average Phase Algorithm}

Because of the $2 \pi$ period of the phase existing in the exponential $e^{j \theta_{j i}}$, taking no account of the adverse effect from the noise, the estimation of the $\theta_{j i}(\omega)$ is

$$
\hat{\theta}_{j i}(\omega)=\theta_{j i}(\omega)-2 k_{j i}(\omega) \pi
$$

where $\hat{\theta}_{j i}(\omega) \in(-\pi, \pi], \quad k_{j i}(\omega)=\{ \pm 1,0\}$. If imposing the estimation value to compensate the signal directly, that is 


$$
\varphi_{i}^{c}(\omega)=\varphi_{i}(\omega)+\frac{1}{L} \sum_{j=0, j \neq i}^{L-1} \hat{\theta}_{j i}(\omega)=\frac{1}{L} \sum_{i=0}^{L-1} \varphi_{i}(\omega)-\frac{2 \pi}{L} \sum_{j=0, j \neq i}^{L-1} k_{j i}(\omega)
$$

We can see clearly that there are two problems due to the phase ambiguity. First, $k_{j i}(\omega)$ is related to the frequency $\omega$, it means that $k_{j i}\left(\omega_{k}\right)$ is probably unequal to the $k_{j i}\left(\omega_{n}\right)$ when $\omega_{k} \neq \omega_{n}$, and the phase of the combined channel will exist in the phenomenon of the phase jump. The issue can be solved by phase unwrapping with the way that whether the phase difference of the close frequency for the channel exceeds the value the $2 \pi / L$ or not. Second, the signals could not co-phase to combine because of $\varphi_{i}^{c}(\omega) \neq \varphi_{j}^{c}(\omega)+2 l \pi, l=\{ \pm 1,0\}$. It is necessary to eliminate the phase ambiguity among the branches. It is easy to co-phase combing adopting the single reference, the relative average phase algorithm is proposed. Without loss of generality, taking the 0th branch as the reference, the phase estimation is $\hat{\theta}_{i 0}(\omega)$. The compensative phase $\hat{\theta}_{i 0}^{\prime}(\omega)$ is zero mean, that is

$$
\hat{\theta}_{i 0}^{\prime}(\omega)=\hat{\theta}_{i 0}(\omega)-\frac{1}{L} \sum_{j=0}^{L-1} \hat{\theta}_{j 0}(\omega)
$$

Therefore the combined channel's phase is

$$
\varphi_{i}^{\prime c}(\omega)=\varphi_{i}(\omega)-\hat{\theta}_{i 0}^{\prime}(\omega)=\varphi^{c}(\omega)+\frac{2 \pi}{L} \sum_{j=1}^{L-1} k_{0 j}(\omega)
$$

It is obvious that the signals with the compensative value $^{\hat{\theta}_{i 0}(\omega)}$ have the same phase center, and can co-phase and add together. Considering the changeless phase excursion do not damage the demodulation performance, thus the combining performance is impossible to degrade due to the phase excursion. Of course, the phase unwrapping should be utilized to combat the phase jump caused by the $k_{0 j}(\omega)$ with the different frequency.

\section{Simulation RESUlts}

In this section, a series of simulations are carried out to study the proposed algorithm's performance. Without loss of generality, the following assumptions and parameters are used. We suppose that carrier frequency offset $\Delta f=0, \mathrm{~L}=4, \mathrm{SNR}=-$ $2 \mathrm{~dB}$ and the channel coefficient expressed in the Equation (13), The initial phases are yielded randomly in the range of $(0 \sim 2 \pi)$. The $g_{T}(t)$ is a square root raised cosine filter with the roll-off factor 0.5 to produce QPSK modulated baseband signal and the symbol period $T=1$ with two samples per symbol. In the demodulation, the symbol space equalization CMA is utilized with the parameter equalizer length $\mathrm{M}=16$ and step size $\delta=0.001$, and the timing synchronization Gardner algorithm is also adopted before the equalizer.

$$
\left\{\begin{array}{l}
c_{1}=[1,0.42-0.48 \mathrm{i}, 0.35-0.13 \mathrm{i}, 0.03+0.27 \mathrm{i}] \\
c_{2}=[-0.23-0.30 \mathrm{i}, 1,0.29-0.03 \mathrm{i},-0.4+0.06 \mathrm{i}] \\
c_{3}=[-0.43+0.01 \mathrm{i}, 0.27+0.29 \mathrm{i}, 1,-0.26+0.14 \mathrm{i}] \\
c_{4}=[0.46-0.46 \mathrm{i}, 0.07-0.15 \mathrm{i}, 1,-0.43-0.25 \mathrm{i}]
\end{array}\right.
$$

As it is described, the SNR of the combined signal becomes greater than the single's. Figure.1 illustrates the amplitudefrequency characteristic of the combined channel and the branch in case of the same noise power density. We can see clearly that the combined channel distortion will be reduced besides improving the ratio of signal to noise (SNR), and it is quite effective for the demodulator. After the time synchronization and the CMA equalizer, figure. 2 shows the mean square error (MSE) curves of the combined signal with the single phase reference and the average phase reference. We can see obviously that the algorithm with the average phase reference has the best performance in term of the MSE curves. As a result, it could improve the phase frequency characteristic and overcome the shortcoming from the fixed single method.

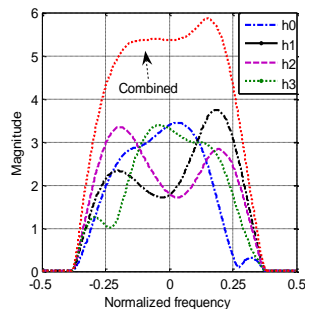

FIGURE I. THE AMPLITUDE-FREQUENCY CHARACTERISTI

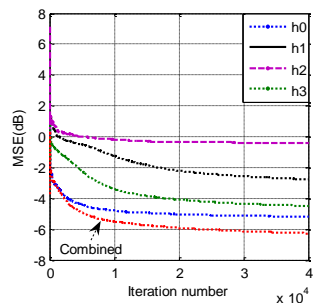

FIGURE II. MSE CURVES WITH THE ITERATION

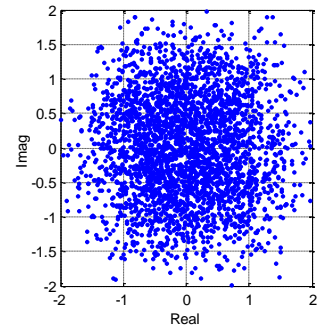

(a) the first signal

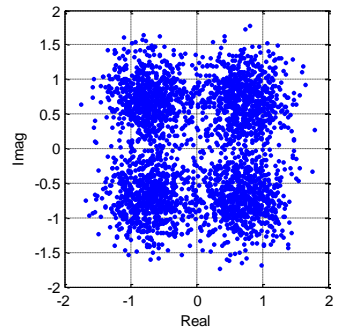

(b) the combined signal
FIGURE III. CONSTELLATION OF THE SIGNALS

Moreover, the signals' constellations are showed in Figre.3. We also can find that the constellation of the combined signal is clearer than the single's, which means the SRE is smaller. Moreover the phenomenon of the lock loss often exists in the demodulator for the single signal because of the multi-path channel and the low SNR, but none for the combined signal. 
According to statistics the SER of the combined signal is 0.031 while the single's is not acquired.

\section{CONCLUSION}

Spectrum combining technique with the average phase algorithm without synchronization is proposed to combat the adverse multi-path in the frequency selective channel at low SNR. The performance of the combining algorithm is not depended on the single reference. Results show that it could improve the amplitude-frequency and phase-frequency characteristic, and it is very usefully for the demodulator with Baud Spaced Equalizer after symbol timing synchronization, on the assumption of the unknown channel.

\section{ACKNOWLEDGMENT}

This work is supported by the National Natural Science Foundation of China (No.61201380). The author would like to thank their colleagues for useful discussions and suggestions.

\section{REFERENCES}

[1] J. G. Proakis, Digital communication, $4^{\text {th }}$ ed., McGraw-Hill, 2001.

[2] P. Balaban and J. Salz, "Optimum diversity combining and equalization in digital data transmission with applications to cellular mobile radioPart II," IEEE Trans. Commun., vol. 40, no. 5, pp. 885-894, 1992.

[3] P. Balaban and J. Salz, "Optimum diversity combining and equalization in digital data transmission with applications to cellular mobile radioPart II,” IEEE Trans. Commun., vol. 40, no. 5, pp. 895-907, 1992.

[4] R.Krenz and K. Wesołowski. "Comparative Study of Space-Diversity Techniques for MLSE Receivers in Mobile Radio,"IEEE Trans. Veh. Technol, vol. 46, no. 3, pp. 653-663, 1997.

[5] M. Clark, "Adaptive frequency-domain equalization and diversity combining for broadband wireless communications," IEEE J. Select. Areas Commun., vol. 16, pp. 1385-1395, Oct. 1998.

[6] Zhou Feng, Wei Wang and Ran Guo, "Method of Space Diversity Frequency Combining Based on Single Carrier Frequency Domain Equalization," in Proc. IEEE ISCIT'2011, pp. 184-187.

[7] Ji Zhong-mei, Yang Hong-sheng and Wang Da-ming, "Synchronization technology and its application in communication", Tsinghua University Press, 2008.

[8] E.R.Rodemich, V.A.Vilnrotter. Digital Combining-Weight Estimation for Broadband Sources using Maximum-Likelihood Estimations [R]. TDA Progress Report 42-116, Jet Propulsion Laboratory Pasadena California, pp.98-109. February 1994.

[9] Shen Cai-yao, Yu Hong-yi and Hu Yun-peng, "An Signal Component Combining Algorithm in Multi-antennas array over the Frequency Selective Channels," Journal of Information Engineering University, vol. 16, pp. 48-53. 2012. 